\title{
The undecidable dynamics generate quantum probabilities
}

\author{
Open Quantum Collaboration*广
}

September 5, 2021

\begin{abstract}
We conjecture a new approach to quantum mechanics that, if confirmed, will explain the wave function from a fundamentally deeper level.
\end{abstract}

keywords: quantum mechanics, dynamical system, undecidability, many-worlds, Everett, wave function

The most updated version of this white paper is available at

https://osf.io/hfc82/download

https://zenodo.org/record/5068737

\section{Introduction}

1. We present two conjectures as an attempt to explain the quantum wave function.

*All authors with their affiliations appear at the end of this white paper.

†Corresponding author: mplobo@uft.edu.br|Open Quantum Collaboration 


\section{Many-worlds}

2. The many-worlds Everettian approach of quantum mechanics (MW) is delightfully explained in this wonderful book [1].

3. It is however a hard pill to swallow.

4. It has indeed one advantage; it deals with the idea that our universe can rely on rules inaccessible from our limited four-dimensional world.

5. I consider (4) a plus side because I often see physicists ruling out very plausible ideas due to inaccessibility grounds.

6. Regarding MW, let's suppose two situations:

(a) the measurement branches the wave function, creating different material worlds;

(b) the measurement branches the wave function, creating different ghostly worlds.

7. By material worlds, I mean ordinary spacetime dimensions with regular matter and energy as we are accustomed to.

8. By ghostly world, I mean a multidimensional mathematical space with exotic forms of matter and energy.

9. Both different material/ghostly worlds are not meant to be accessible to us.

10. If MW proves to be correct, I would bet on (6.b).

\section{The undecidability theorem}

11. There exists unpredictable algorithms. [1,2] 


\section{Assumptions}

12. The following conjectures are meant to hold in the Planck scale.

13. Conjecture 1: Quantum undecidability $(Q U)$ Quantum fluctuations are originated by undecidable mathematical algorithms.

14. Conjecture 2: Quantum dynamics $(Q D)$ Quantum probabilities are originated by spacetime's dynamical system.

15. $Q U$ leads to the indeterminacies that are intrinsic to the quantum realm such as, for instance, not knowing precisely the exact position being measured.

16. $Q D$ is given deterministically by an iterated mathematical function.

\section{Dynamical systems}

17. Consider a discrete dynamical system with the orbit

$$
f\left(x_{0}\right) \rightarrow f\left(f\left(x_{0}\right)\right) \rightarrow f\left(f\left(f\left(x_{0}\right)\right)\right) \rightarrow \ldots,
$$

where $x_{0}$ is the initial condition.

18. If spacetime is discrete, then it is natural to suppose it is described by discrete dynamical systems.

19. The Lorentzian symmetry of spacetime suggests either both space and time are discrete or likewise both are continuous.

20. Other combinations arise, such as discrete time and continuous space, and continuous time and discrete space, but I believe they are seemingly improbable due to (19). 


\section{Undecidability in quantum dynamics}

21. In the Planck scale, due to the quantum fluctuations (uncertainty principle), one cannot determine exactly a point $x_{0}$ in space.

22. (13) states that nature operates with undecidable mathematical algorithms beforehand, leading to initial positions $x_{0}$ that are intrinsically unpredictable.

23. Then a deterministic function iterates $x_{0}$ and produces the probabilities of quantum states.

24. Each orbit of the mathematical dynamical system depicts the collapse of the corresponding quantum states $[3,4]$.

\section{Final Remarks}

25. According to our conjectures, quantum spacetime is generated by undecidable mathematical algorithms, which then evolve as a discrete/continuous dynamical system.

26. Whether the mathematical dynamical system describing the quantum spacetime is discrete or continuous would depend upon the discreteness of spacetime itself.

\section{Open Invitation}

Review, add content, and co-author this white paper $[5,6]$.

Join the Open Quantum Collaboration.

Send your contribution to mplobo@uft.edu.br. 


\section{Open Science}

The latex file for this white paper together with other supplementary files are available in $[7,8]$.

\section{How to cite this paper?}

https://doi.org/10.31219/osf .io/hfc82

https://zenodo.org/record/5068737

\section{Acknowledgements}

+ Center for Open Science https://cos.io

+ Open Science Framework https://osf .io

+ Zenodo https://zenodo.org

\section{Agreement}

All authors agree with [6].

\section{License}

CC-By Attribution 4.0 International [9]

\section{References}

[1] Carroll, Sean. Something deeply hidden: quantum worlds and the emergence of spacetime. Dutton, 2019. 
[2] Wikipedia. Undecidable problem.

https://en.wikipedia.org/wiki/Undecidable_problem

[3] Lobo, Matheus P. "The Logistics of Quantum Spacetime." OSF Preprints, 10 May 2021.

https://doi.org/10.31219/osf.io/s2dnt

[4] Lobo, Matheus P. "Time Is a Discrete Dynamical System." OSF Preprints, 21 May 2021.

https://doi.org/10.31219/osf .io/8f4yg

[5] Lobo, Matheus P. "Microarticles." OSF Preprints, 28 Oct. 2019. https://doi.org/10.31219/osf .io/ejrct

[6] Lobo, Matheus P. "Simple Guidelines for Authors: Open Journal of Mathematics and Physics." OSF Preprints, 15 Nov. 2019.

https://doi.org/10.31219/osf .io/fk836

[7] Lobo, Matheus P. "Open Journal of Mathematics and Physics (OJMP)." OSF, 21 Apr. 2020. https://osf .io/6hzyp/files

[8] https://zenodo.org/record/5068737

[9] CC. Creative Commons. CC-By Attribution 4.0 International. https://creativecommons.org/licenses/by/4.0

\section{The Open Quantum Collaboration}

Matheus Pereira Lobo (lead author, mplobo@uft.edu.br) $)^{1,2}$ https://orcid.org/0000-0003-4554-1372

${ }^{1}$ Federal University of Tocantins (Brazil)

${ }^{2}$ Universidade Aberta (UAb, Portugal) 